\title{
Dural sinus obstruction following head injury: a diagnostic and clinical study
}

\author{
*Mony Benifla, MD,' Uri Yoel, MD,,,3 Israel Melamed, MD, ${ }^{2,4}$ Vladimir Merkin, MD, ${ }^{2,4}$ \\ Avi Cohen, MD, ${ }^{2,4}$ and Ilan Shelef, MD ${ }^{5}$
}

\begin{abstract}
${ }^{1}$ Department of Neurosurgery, Hadassah Medical Center, Jerusalem; ${ }^{2}$ Faculty of Health Sciences, Ben-Gurion University of the Negev, Beer-Sheva; and Departments of ${ }^{3}$ nternal Medicine, ${ }^{4}$ Neurosurgery, and ${ }^{5}$ Radiology, Soroka University Medical Center, Beer-Sheva, Israel
\end{abstract}

OBJECTIVE The aim of this study is to describe the clinical characteristics of patients with skull fracture adjacent to a dural venous sinus (DVS) and evaluate the role of CT venography (CTV) in the diagnosis of the effect of this fracture on the involved DVS.

METHODS The study comprised patients with vault or skull base fracture adjacent to a DVS who were admitted to 1 medical center during a 2-year period. The medical records were reviewed for the clinical and radiographic characteristics. All patients had undergone CTV to evaluate potential DVS pathology. The clinical and radiological findings of the patients with DVS pathology were compared with those of the patients with normal DVS. The groups were compared using the chi-square and t-tests for categorical and continuous data, respectively. The potential risk for poor outcome among the patients with DVS pathology was also investigated.

RESULTS Of 434 patients with skull fractures, 41 (9.4\%) had fractures adjacent to a DVS. DVS pathology was detected in $51 \%$ of patients (21 of 41 patients). For 9 (43\%) patients, obstruction was extraluminal without thrombosis, and 12 (57\%) patients had dural sinus thrombosis (DST). In patients with a positive-CTV scan, the severity of injury according to the Glasgow Coma Scale score at presentation was correlated with the presence of DST $(p=0.007)$. The sensitivity of noncontrast CT (NCCT) for DVS involvement was 38\% among the patients with positive-CTV scans. For patients with DVS pathology, poor outcome was correlated with DST (intraluminal), rather than extraluminal obstruction without thrombosis $(p=0.02)$, and superior sagittal sinus (SSS) involvement $(p=0.05)$.

CONCLUSIONS NCCT is not sensitive enough to detect DVS obstruction in patients with skull fracture adjacent to a DVS, and CTV should be performed in order to rule it out. A correlation was found between the severity of injury and the presence of DST, rather than extraluminal obstruction. The authors' findings suggest that DST and SSS involvement may be indicators of poor outcome in patients with skull fracture adjacent to DVS.

http://thejns.org/doi/abs/10.3171/2016.3.PEDS15690

KEY WORDS computed tomography venography; dural sinus thrombosis; head injury; spiral computed tomography; trauma

$\Lambda$ STUDY from over 2 decades ago reported the incidence of traumatic dural sinus thrombosis (DST) as $4 \%$ with penetrating head trauma and rare with closed head injury. ${ }^{25}$ However, more recently, DST has been documented in $6 \%$ to $50 \%$ of traumatic skull fractures. ${ }^{6,29}$ Traumatic DST presents with a wide and nonspecific spectrum of signs and symptoms, the most common being a decreased level of consciousness, headache, nausea and vomiting, papilledema, visual abnormality, gait ataxia, seizures, and focal neurological deficits. ${ }^{1,3,9,10,16,19,26,30,33-36}$ The exact chain of events that results in thrombosis after head trauma is unclear. Bone fragments, sinus obstruction, or epidural hematoma displacing the adjacent dural venous sinus (DVS) may form an obstruction or turbulent flow, which in turn induces thrombus formation. . $^{3,11,16,36}$

Noncontrast CT (NCCT) is usually the first urgent

ABBREVIATIONS CTV = CT venography; DST = dural sinus thrombosis; DVS = dural venous sinus; $\mathrm{GCS}=$ Glasgow Coma Scale; $\mathrm{GOS}=\mathrm{Glasgow}$ Outcome Scale; ICP = intracranial pressure; NCCT = noncontrast CT; SSS = superior sagittal sinus; $\mathrm{TS}=$ transverse sinus.

SUBMITTED November 21, 2015. ACCEPTED March 4, 2016.

INCLUDE WHEN CITING Published online May 6, 2016; DOI: 10.3171/2016.3.PEDS15690.

* Drs. Benifla and Yoel contributed equally to this work. 
imaging study performed for head injury patients., ${ }^{2,4,14,16}$ Findings suggestive of DST on NCCT scan include highdensity clots in the cortical veins and DVS, which are termed the "cord" and "dense triangle" sign, respectively. ${ }^{2}$ In patients after head injury, a fracture crossing over a DVS or an adjacent hematoma seen on NCCT scan may indicate thrombosis. ${ }^{3,6,8,16,25}$ More sensitive and specific is the "empty delta" sign, which is visible after contrast injection, and represents a filling defect within a DVS., ${ }^{2,32}$ The use of high-speed CT scanners enables scanning during peak venous enhancement and thus increases sensitivity to obstructions within intracranial venous structures. ${ }^{16,20} \mathrm{Al}$ though very few systematic studies have assessed diagnostic strategies against a gold standard, both gadolinium-enhanced MR techniques and multislice contrast-enhanced CT (i.e., CT venography) are considered reliable techniques for the diagnosis of DST. ${ }^{23,24}$ Nonetheless, in the clinical setting of the acutely injured patient, CT venography (CTV) has some obvious practical advantages over MR techniques due to its availability, simplified screening process, and the short time of the procedure. ${ }^{16,27,28}$

While the treatment of choice in adults with nontraumatic DST is anticoagulation therapy, ${ }^{5,13,14}$ no specific therapy directed against the thrombotic process is generally recommended for traumatic DST, especially with mild injury. ${ }^{1,15,16,19,35-37}$ However, surgical treatment or endovascular intervention is a safe and effective approach, especially for patients with severely elevated intracranial pressure (ICP) syndrome whose conditions are refrac-

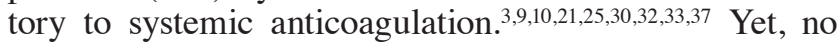
large-series data dealing specifically with the outcome of traumatic DVS obstruction with or without thrombosis are available.

The aims of this study were to describe the clinical and radiological characteristics of patients with skull fracture adjacent to DVS (i.e., crossed over the sinus) in order to assess treatment and outcomes and evaluate the role of CTV in the diagnosis of DVS pathology in these patients.

\section{Methods \\ Patients}

The cohort comprised patients who were admitted to Soroka Medical Center and had skull fracture crossing a DVS, as detected by NCCT scans. During a 2-year period, all patients had undergone evaluation for DVS involvement using CTV according to our clinical protocol. We collected patient files after cross-matching data from 3 sources, 2 of which were computerized (the ICD-9 system for discharge diagnosis and the database of the Department of Radiology). This procedure helped reduce ascertainment bias. This study was approved by the local institutional review board at Soroka Medical Center.

Patient medical records were reviewed and analyzed for demographic parameters, clinical features, radiographic findings, treatment, and outcome. Injury severity was classified, using the Glasgow Coma Scale (GCS) as mild (GCS Score 14-15), moderate (GCS Score 9-13), or severe (GCS Score 3-8). ${ }^{20}$ Treatment was defined as conservative (e.g., observation), surgical, or medical (e.g., anticoagulants). All patients were followed for at least 1 year from the injury, and the mean follow-up time was 9.3 months. Outcomes were evaluated using the Glasgow Outcome Scale score (GOS Score 5, good recovery and mild disability; GOS Score 4, moderate disability; GOS Score 3, severe disability; GOS Score 2, persistent vegetative state; or GOS Score 1, death) ${ }^{31}$ Unfavorable outcome was defined as GOS Score 1-3, and favorable outcome was defined as GOS Score 4-5.

\section{Imaging Techniques and Interpretation}

At the emergency room of our medical center, the imaging protocols for patients with head injury include CT and CTV (if needed). Subsequent imaging may include MR and MR venography, especially for pediatric patients if they are able to withstand it. The NCCT scans were performed using an axial technique with 120 to $140 \mathrm{kVp}, 170 \mathrm{~mA}$, and 5.0-mm-thick section reconstructions for the standard head algorithm and 2.5-mm-thick section reconstructions for the head-bone algorithm. The NCCT films were screened for the following parameters: the location of the fracture, the appearances and locations of the hematomas (epidural, subdural, or subarachnoid) or parenchymal hemorrhages, the presence of contusions, sizes of the ventricles, signs of white matter edema and intracranial air bubbles, and the presence of the dense triangle or cord sign.

CTV was used to illustrate DVS involvement in patients with head trauma. CTV was performed on the head from the base of the C-1 vertebra to the vertex after a fixed 60 -second delay between the onset of contrast material injection and the start of scanning. A 65- to 85-ml volume of nonionic iodinated contrast material was injected into the antecubital vein at 4 to $5 \mathrm{ml} / \mathrm{second}$ using a power injector. In children younger than 2 years, the material was injected manually by a trained intensive care unit physician. The following scanning and reconstruction parameters were used: pitch of 0.5 ; collimation of $1.25 \mathrm{~mm}$; maximal tube current of $250 \mathrm{~mA}$; tube voltage of $120 \mathrm{kVp}$; field of view of $22 \mathrm{~cm}$; and $1.25-\mathrm{mm}$-thick section reconstruction. Standard maximum-intensity projection images of the major intracranial venous structures were created. Threedimensional reformation with the transparency technique was performed to enable correlating the lesion in the sinus with the abnormal bone.

Both CTV and NCCT studies are components of our routine clinical protocol. For the current study, the same trained, experienced neuroradiologist, who was blinded to the clinical status upon arrival and outcomes, reviewed the NCCT and CTV studies. The following findings were tabulated: empty delta sign; gyral enhancement; tentorial enhancement; and the presence, degree, and type of a DVS obstruction. When such a distortion was found, it was classified according to its degree as complete or partial, and its type was classified as extraluminal (epidural hematoma or depressed skull fracture that displaces the adjacent DVS without signs of a filling defect within a DVS lumen), intraluminal due to a thrombus within a DVS (DST), or as a combination of these 2 findings.

\section{Statistical Analyses}

The following variables were examined: age; sex; mech- 
anism of injury; severity of injury; clinical manifestations; radiological findings; treatment; and outcome. Statistical univariate analyses were performed with the chi-square test for categorical data and the Student t-test for continuous data. Potentially important differences between patients with an obstructed DVS lumen (partial or complete), as established by CTV (the positive-CTV group), and those with normal DVS on the CTV scan (the negativeCTV group) were examined for each of the variables noted above. In addition, patients in the positive-CTV group were evaluated for possible correlations between demographic, clinical, and radiological parameters and outcome. Statistically significant results were defined as a p value $<0.05$. Due to a nonnormal distribution, we used the Spearman correlation test to determine the strength of the correlations and the Kruskal-Wallis test to compare all 3 groups (Tables 1 and 2). For correlations between groups with small numbers of individuals, we used the nonparametric Fisher's exact test. Statistical analysis was performed with IBM SPSS (version 20). All $p$ values were 2-sided, and $p<$ 0.05 was considered statistically significant.

\section{Results}

\section{Demographic and Clinical Characteristics}

During the study period, 434 admitted patients were identified with skull fractures. Of these, 41 (9.4\%) patients had fractures adjacent to a DVS and were further evaluated using CTV. In 21 patients, some degree of distortion of a DVS was found (the positive-CTV group). These patients comprised $4.8 \%$ of all patients with skull fracture and $51 \%$ of those who underwent CTV. Fifteen (71\%) pa-

TABLE 1. Selected clinical characteristics of 41 patients with a fracture line adjacent to a DVS*

\begin{tabular}{|c|c|c|c|}
\hline Clinical Characteristic & Total & $\begin{array}{l}\text { Positive } \\
\text { CTV }\end{array}$ & $\begin{array}{c}\text { Negative } \\
\text { CTV }\end{array}$ \\
\hline No. of patients & 41 & 21 & 20 \\
\hline \multicolumn{4}{|l|}{ Mechanism of injury } \\
\hline Road accident & $19(46)$ & $6(29)$ & $13(65)$ \\
\hline Fall from height & $13(32)$ & $8(38)$ & $5(25)$ \\
\hline Falling object & $4(10)$ & $2(9)$ & $2(10)$ \\
\hline Other & $5(12)$ & $5(24)$ & 0 \\
\hline \multicolumn{4}{|l|}{ GCS score on arrival } \\
\hline 14-15 (mild) & $19(46)$ & $9(43)$ & $10(50)$ \\
\hline 9-13 (moderate) & $11(27)$ & $5(24)$ & $6(30)$ \\
\hline 3-8 (severe) & $11(27)$ & $7(33)$ & $4(20)$ \\
\hline \multicolumn{4}{|l|}{ Clinical manifestations } \\
\hline Loss of consciousness & $16(39)$ & $9(43)$ & $7(35)$ \\
\hline Altered consciousness & $14(34)$ & $8(38)$ & $7(35)$ \\
\hline Headache & $9(22)$ & $6(29)$ & $3(15)$ \\
\hline Vomiting & $10(24)$ & $6(29)$ & $4(20)$ \\
\hline Seizures & $2(5)$ & $2(9)$ & 0 \\
\hline Dilated pupils & $6(15)$ & $5(24)$ & $1(5)$ \\
\hline Gaze deviation & $1(2)$ & $1(5)$ & 0 \\
\hline Focal signs & $4(10)$ & $2(9)$ & $2(10)$ \\
\hline
\end{tabular}

* Data are shown as number of patients (\%).
TABLE 2. Radiological findings in 41 patients with a fracture adjacent to a DVS, as established by NCCT and CTV scans according to the degree of the obstruction*

\begin{tabular}{lllll}
\hline & \multicolumn{3}{c}{ Degree of Obstruction } & $p$ \\
\cline { 2 - 4 } \multicolumn{1}{c}{ Variable } & None & Partial & Complete & Value \\
\hline No. of patients & 20 & 6 & 15 & \\
\hline NCCT findings & & & & \\
\hline Hematoma & $6(30)$ & $4(67)$ & $8(53)$ & 0.08 \\
\hline Air bubbles & $9(45)$ & $1(17)$ & $5(33)$ & 0.27 \\
\hline Dense triangle sign & $1(5)$ & $1(17)$ & $7(47)$ & 0.02 \\
\hline Contusion & $5(25)$ & $2(33)$ & $4(27)$ & 0.80 \\
\hline Parenchymal hemorrhage & $4(20)$ & $1(17)$ & $3(20)$ & \\
\hline White matter edema & $4(20)$ & $3(50)$ & $9(60)$ & 0.01 \\
\hline CTV finding & & & & \\
\hline Empty delta sign & 0 & $2(33)$ & $10(67)$ & 0.33 \\
\hline Gyral enhancement & 0 & $1(17)$ & $4(27)$ & \\
\hline Tentorial enhancement & 0 & $2(33)$ & $5(33)$ & \\
\hline
\end{tabular}

* When a patient had more than 1 sinus involved, the most severe obstruction was considered.

† For NCCT findings, the $p$ values (2-sided) compare the patients without evidence of obstruction and patients with some degree of obstruction (both partial and complete). For the CTV findings, the $p$ values (2-sided) compare partial and complete obstruction.

$\ddagger$ Epidural or subdural hematoma adjacent to the relevant dural sinus.

tients were male, 6 (29\%) patients were female, the age range was 4 months to 86 years, the median age was 13 years, and the mean age was 17.6 years. In the other 20 patients, no distortion of the DVS system was established by CTV (the negative-CTV group). Sixteen (80\%) patients were male, $4(20 \%)$ patients were female, the age range was 1 to 48 years (mean age 14.65 years; $p=0.61$ ), and 13 of the patients with normal sinuses $(65 \%)$ were younger than 16 years.

A higher proportion of patients in the positive-CTV group had severe injury (GCS Score 3-8) than patients in the negative-CTV group: 7 (33\%) patients versus 4 (20\%) patients, respectively. Moreover, 5 of 7 severely injured patients (71\%) in the positive-CTV group arrived with GCS scores $\leq 5$, whereas only 1 of 4 severely injured patients in the negative-CTV group (25\%) had GCS scores $\leq 5$. Severe clinical manifestations were more prevalent in the positive- than in the negative-CTV group (Table 1). We used the chi-square test to compare the positive- and negative-CTV groups. Importantly, within the positive-CTV group, these signs were more common among severely injured (6 of 7 patients [86\%]) than mildly to moderately injured patients ( 1 of 14 patients [7\%]; $\mathrm{p}=0.001$ ). In addition, most of the mildly to moderately injured patients in the positive-CTV group (9 of 14 patients [64\%]) were defined as harboring pure extraluminal lesions, whereas all severely injured patients (7 of 7 patients) were found to have a component of DST (intraluminal) on their CTV $(\mathrm{p}=0.007)$.

\section{Radiological Findings}

Findings suggestive of the presence of a pathological DVS on the NCCT scans (except from a fracture line ad- 
jacent to a DVS) were either direct (dense triangle sign suspected to be intraluminal DST) or indirect (hematoma or air bubbles over the course of the DVS suspected to be an extraluminal lesion). The most statistically significant finding was white matter edema $(\mathrm{p}<0.01)$.

In the positive-CTV group, the dense triangle sign on NCCT was detected in only 8 patients (38\%). Indirect suggestive findings were more common and recognized in 14 patients (67\%) (Table 2). Still, in these 8 patients (53\%), of those with an established obstruction of a DVS, the initial NCCT scan showed no indication of such an obstruction. Of the patients with normal NCCT, 7 patients were children younger than 16 years. All of these patients had a parietal, biparietal, occipital, or combined fracture. A fall from a height was the mechanism of injury in 4 patients and road accident in 3 patients. For 1 child, abuse was suspected. A GCS score of 15 was observed in 4 patients in the NCCT group at the time of admission: 2 patients were admitted with a GCS score of 12, and 2 patients as GCS score of 8 . The GOS score was 5 in 6 children, 4 in 1 child, and 3 in another child. Importantly, of these 8 patients, 4 were found to have complete obstruction on CTV (2 patients with an extraluminal obstruction and 2 with a large DST).

Within the positive-CTV group, in $9(43 \%)$ patients the type of distortion was extraluminal with no signs of a filling defect in CTV. In 6 of these patients, the cause of this distortion was an epidural hematoma that displaced the adjacent DVS, and in 3 patients the cause was a bone fragment from a depressed skull fracture. In 4 patients (19\%), filling defects were observed on CTV without any signs of extraluminal sinus compression. Eight other patients (38\%) were found to have both types of distortion. Figure 1 demonstrates the relative involvement of the DVS. Figures 2 and 3 demonstrate the importance of CTV in cases in which the routine NCCT scan seems to be unremarkable.

\section{Treatment}

Conservative treatment-i.e., bed rest, hydration, and close observation-was initiated in 18 patients. Three patients were operated on soon after they presented to our service, for evacuation of either an epidural or subdural hematoma that was fully obstructing the sinus. ICP monitoring was performed in 6 patients according to common practice indications. Another 3 patients had initial clinical improvement and then deteriorated due to occlusion of the dural sinuses. One of these patients was a 14-year-old girl who arrived to the hospital with a GCS score of 12 after a road accident. Initial CT showed no abnormal findings other than a fracture adjacent to the right transverse sinus (TS). She gradually improved, and on Day 4 she complained of severe headache and vomiting. On examination, this patient had cranial nerve VI palsy and papilledema. CTV showed thrombosis of the right transverse-sigmoid sinus (Fig. 3C). Anticoagulant therapy was started (80 U/ day low-molecular-weight heparin), and she significantly improved over time. Six months after the injury, she fully recovered (GOS Score 5). Follow-up CTV revealed recanalization through the obstructed sinus.

The second of the 3 patients was a 22-year-old man

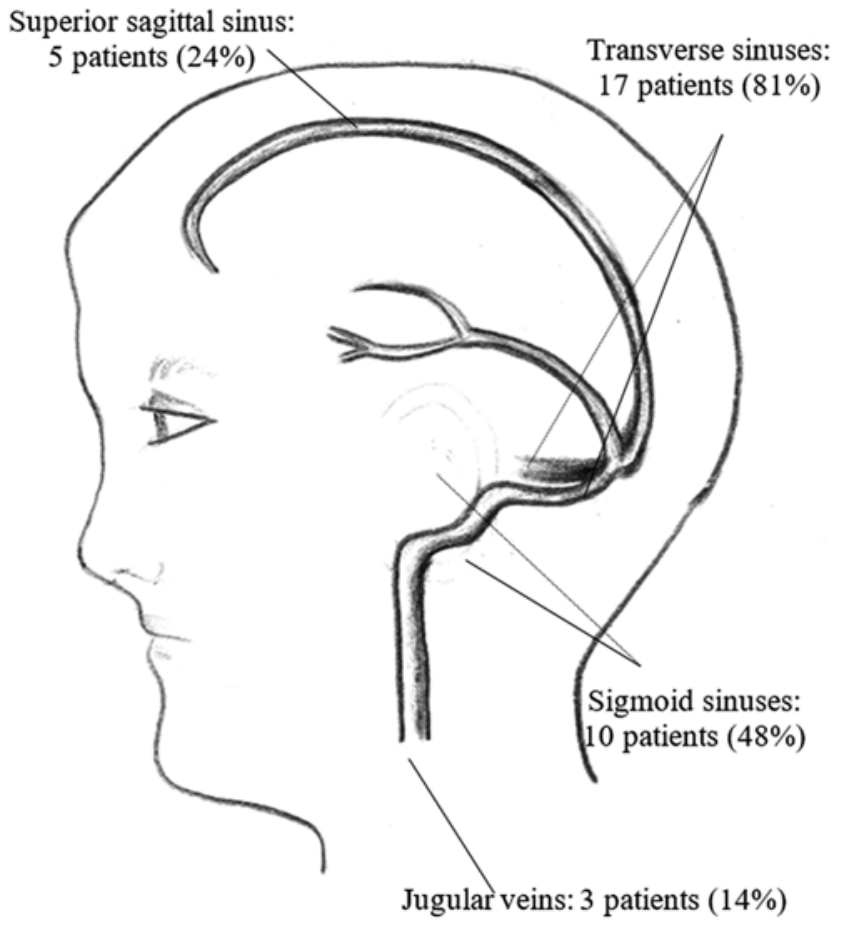

FIG. 1. Schematic lateral view of the cerebral sinovenous system. The structures most susceptible to dural sinus occlusion with or without thrombosis in the patients in the positive postcontrast spiral CT scan group are shown. The relative frequencies are given in parentheses. Multiple sinus involvement was found in 12 patients (57\%), and in 3 patients the thrombus extended from the TS to the jugular vein. Copyright Ilan Shelef. Published with permission.

who arrived with a GCS score of 6 after a road accident. The initial CT scan revealed a depressed fracture over the superior sagittal sinus (SSS) and an epidural hematoma. An urgent craniotomy was performed to evacuate the hematoma and elevate the depressed fracture. During surgery, tears in the SSS were identified and sutured to prevent bleeding. An ICP monitor was inserted and was medically controlled for 4 days. On Day 5, ICP was elevated significantly with no medical control. The patient still had a GCS score of 6 and his pupils were normal, but the first signs of papilledema (the loss of the venous pulsations and a blurred temporal border) were seen. CTV showed sinus vein thrombosis of the SSS. Although this patient still had intraparenchymal contusions and we were aware of the risk of secondary bleeding, a decision was made to commence anticoagulant therapy (1000 U/hour heparin without a loading dose). Six hours after the initiation of heparin and every 24 hours, CT scanning was performed to exclude secondary bleeding. Three days later, the ICP was controlled again with no more signs of sinus reflow. The patient gradually improved, the ICP monitor device was removed 10 days after the injury, and heparin was switched to low-molecular-weight heparin (enoxaparin). Six months later, the patient had a GOS score of 3. Recanalization of the involved DVS was noticed on a follow-up CTV.

The third patient-a 3-year-old boy who fell from a 6-m height-arrived at the emergency department with a 

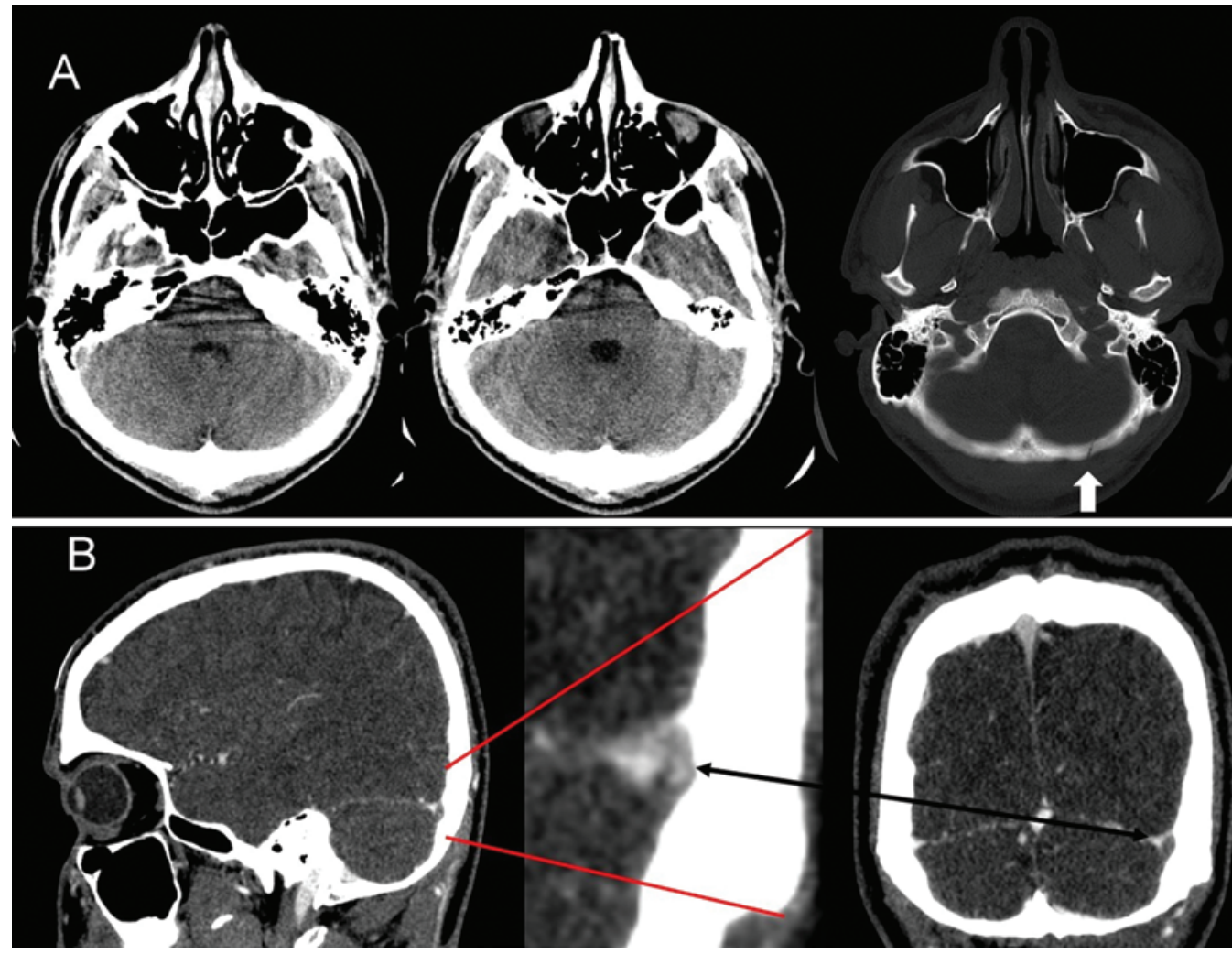

FIG. 2. A: An unremarkable brain noncontrast CT scan of a patient with a petrous bone linear fracture (the thick arrow indicates the bone window) adjacent to the left sigmoid sinus. B: CTV demonstrating an epidural hematoma (black arrow) adjacent to the left sigmoid sinus. The lumen is narrowed by the hematoma. The lines indicate the enlarged image of the sinus area. Figure is available in color online only.

GCS score of 4 and with fully dilated pupils. Initial NCCT revealed multiple brain contusions, massive brain edema, and a longitudinal fracture running down from the right parietal bone to the left side of the skull, all the way down to the occipital bone and crossing the SSS and left TS. Due to the hemorrhages, it was decided not to start anticoagulant therapy. Due to lung and abdominal injuries, he became hemodynamically unstable, developed disseminated intravascular coagulation, and eventually died within 48 hours from multiorgan failure.

\section{Outcome}

In both the positive- and negative-CTV groups, 1 patient died during admission. An unfavorable outcome (GOS Score 1-3) was defined at the end of the clinical follow-up period (1 year) in $3(15 \%)$ and $6(29 \%)$ patients in the negative- and positive-CTV groups, respectively ( $\mathrm{p}$ $=0.45$ ). Of the 6 patients with an unfavorable outcome in the positive-CTV group, 5 (83\%) arrived with GCS scores $\leq 9$ and had multiple intraparenchymal injuries, including edema, contusions, and epidural or subdural hemorrhage. Of these 6 patients, 1 patient died due to severe and diffuse axonal injury and parenchymal hemorrhagic contusion (GOS Score 1), 1 patient was discharged in a vegetative state (GOS Score 2), and 4 patients had severe neurological deficits (GOS Score 3).

We used the nonparametric Fisher's exact test and found a correlation between outcome and the degree of obstruc- tion and type of distortion. Forty percent of patients with complete obstruction had unfavorable outcomes, whereas all patients with partial obstruction were discharged with a favorable outcome $(p=0.12)$. Likewise, when the obstruction included a component of (intraluminal) DST, an unfavorable outcome was observed in $50 \%$ of patients, while all patients with pure extraluminal obstruction had a favorable outcome $(\mathrm{p}=0.02)$. Dominant involvement of a central DVS (e.g., SSS, confluence of sinuses) was also associated with an unfavorable outcome $(\mathrm{p}=0.05$; Table 3$)$.

\section{Discussion}

A number of large multicenter studies involving DST have been published. ${ }^{712,29}$ However, only a small number of trials and case reports dealing specifically with posttraumatic DST are available in the English-language literature. Our results of 21 patients - all with closed head injury and some degree of obstruction in a DVS-indicate that this disturbance is not uncommon among the patients admitted to our institution with a skull fracture: the incidence rate was $4.8 \%$. Moreover, among those patients with a fracture line adjacent to a DVS, $51 \%$ were found to have some degree of obstruction within their DVS system. These findings correlate well with the results of other studies. ${ }^{6,29}$

No specific symptom or sign among the patients in the positive-CTV group was found to be an indicator of the presence of DVS obstruction. Furthermore, all symptoms 

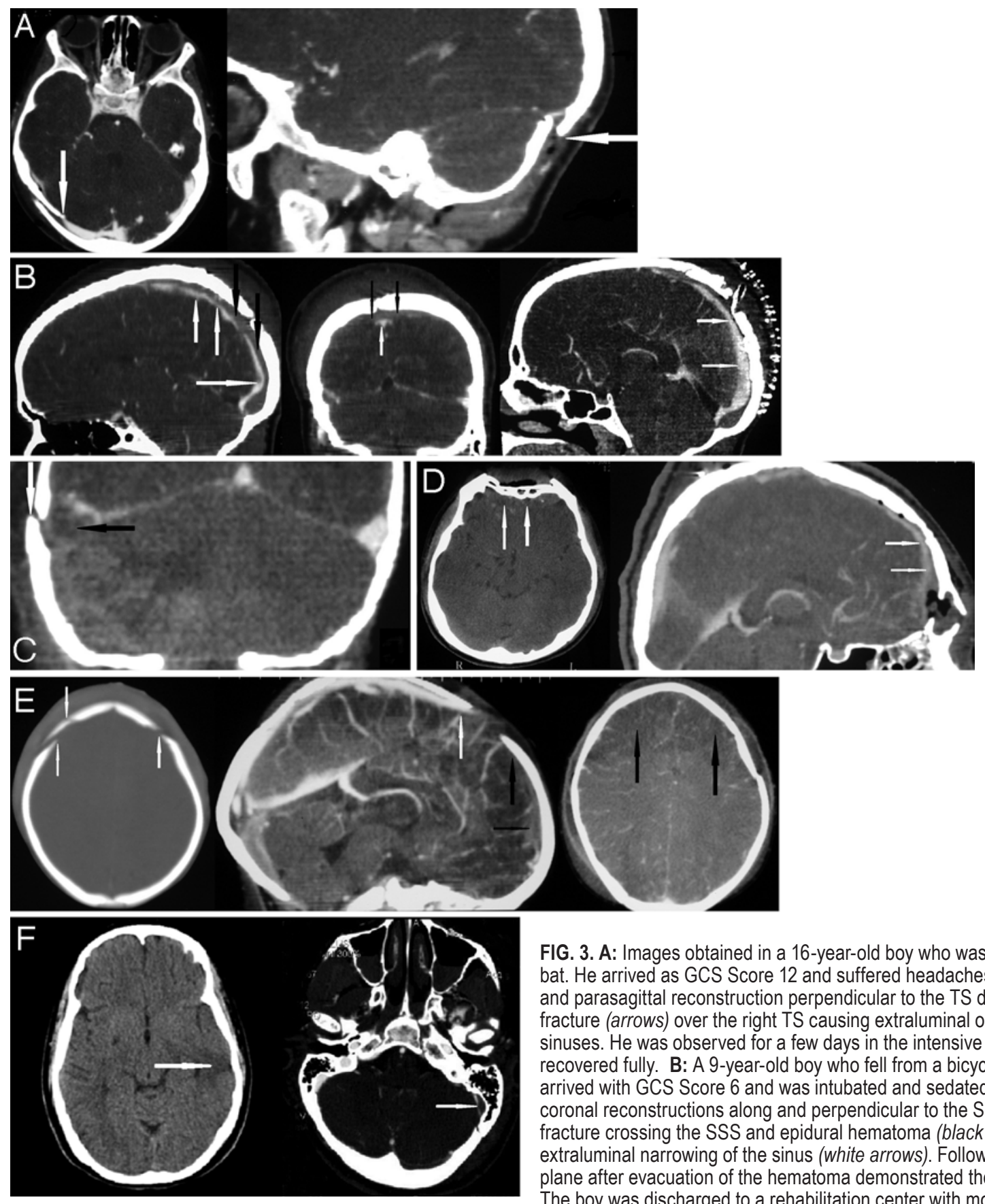

FIG. 3. A: Images obtained in a 16 -year-old boy who was assaulted with a baseball bat. He arrived as GCS Score 12 and suffered headaches and vomiting. CTV axial and parasagittal reconstruction perpendicular to the TS demonstrate the depressed fracture (arrows) over the right TS causing extraluminal obstruction of the dural sinuses. He was observed for a few days in the intensive care unit and with time recovered fully. B: A 9-year-old boy who fell from a bicycle without a helmet. $\mathrm{He}$ arrived with GCS Score 6 and was intubated and sedated. CTV with sagittal and coronal reconstructions along and perpendicular to the SSS demonstrate a linear fracture crossing the SSS and epidural hematoma (black arrows). This resulted in extraluminal narrowing of the sinus (white arrows). Follow-up CTV in the sagittal plane after evacuation of the hematoma demonstrated the reopening of the SSS. The boy was discharged to a rehabilitation center with motor dysphasia, nystagmus, and right hemiparesis. GOS at 6 months after the injury was 3. C: CTV obtained in a 14-year-old girl after a road accident. The initial CT showed a fracture adjacent to the right TS. On Day 4, she developed a headache and vomiting, cranial nerve VI palsy, and papilledema. The coronal reconstruction reveals a minimally depressed fracture adjacent to the right TS and the empty delta sign secondary to the dural sinus thrombosis (arrows). The patient was treated with low-molecular-weight heparin and recovered fully. D: Axial NCCT demonstrating a depressed fracture obliterating the SSS (white arrows on the left image). This 18-year-old male patient arrived as GCS Score 14 after a road accident. He was confused and had obvious rhinorrhea. A bifrontal craniotomy was performed. After remodeling of the fracture and the skull base, sagittal reconstructed CTV demonstrated a reopening of the SSS (white arrows in the right image). The patient recovered fully with GOS 5. E: A 3-year-old boy who fell from a height and arrived as GCS Score 4 with dilated pupils and multitrauma. Unfortunately, this child died of multiorgan failure. Axial NCCT demonstrated a linear fracture (white arrows in the left image) adjacent to the coronal suture crossing the SSS. Sagittal reconstructed CTV demonstrated the complete abrupt obstruction of the anterior part of the SSS due to thrombosis (white arrows in the middle image) and reduced enhancement of both frontal lobes as shown by the black arrows. F: A 40-year-old man who had a minor head injury due to a fall. He suffered increasing headaches over a few days and had no neurological deficit upon arrival to the emergency department. NCCT revealed a low-density area in the posterior aspect of the left temporal lobe involving both the white and gray matter (left). Follow-up CTV demonstrates a filling defect within the sigmoid sinus due to thrombosis (right). 
TABLE 3. Outcomes of 21 patients with any degree of obstruction on the CTV scan according to the selected parameters

\begin{tabular}{|c|c|c|c|c|}
\hline Variable & Total & $\begin{array}{c}\text { GOS } \\
\text { Score 1-3 }\end{array}$ & $\begin{array}{c}\text { GOS } \\
\text { Score 4-5 }\end{array}$ & $\begin{array}{c}p \\
\text { Value }^{*}\end{array}$ \\
\hline \multicolumn{5}{|l|}{ Demographic parameters } \\
\hline Age $\leq 5$ yrs & 8 & $1(12)$ & $7(88)$ & 0.34 \\
\hline Age $>5$ yrs & 13 & $5(38)$ & $8(62)$ & \\
\hline \multicolumn{5}{|l|}{ Mechanism of injury $\dagger$} \\
\hline Road accident & 6 & $2(33)$ & $4(67)$ & 0.08 \\
\hline Damage from falling object & 2 & $2(100)$ & 0 & \\
\hline Fall from height & 8 & 0 & $8(100)$ & \\
\hline Other & 5 & $2(40)$ & $3(60)$ & \\
\hline \multicolumn{5}{|l|}{ Severity of injury } \\
\hline GCS Score 9-15 on arrival & 14 & $2(14)$ & $12(86)$ & 0.12 \\
\hline GCS Score 3-8 on arrival & 7 & $4(57)$ & $3(43)$ & \\
\hline \multicolumn{5}{|l|}{ Radiological parameters } \\
\hline \multicolumn{5}{|l|}{ Degree of obstruction $\ddagger$} \\
\hline Complete & 15 & $6(40)$ & $9(60)$ & 0.12 \\
\hline Partial & 6 & 0 & $6(100)$ & \\
\hline \multicolumn{5}{|l|}{ Mechanism of obstruction§ } \\
\hline Pure extraluminal lesion & 9 & 0 & $9(100)$ & 0.02 \\
\hline Thrombus or both & 12 & $6(50)$ & $6(50)$ & \\
\hline Sinus involvement & & & & 0.05 \\
\hline Lateral system & 17 & $3(18)$ & $14(82)$ & \\
\hline Central system & 4 & $3(75)$ & $1(25)$ & \\
\hline
\end{tabular}

* The $p$ values (2-sided) are for comparisons between favorable and unfavorable outcomes according to selected demographic, clinical, and radiological parameters.

$\dagger$ Road accident together with injury due to a falling object were compared against falling from height for possible differences in outcome.

$\ddagger$ When a patient had more than 1 sinus involved, the most severe obstruction was considered.

$\S$ The mechanism of obstruction was determined according to the interpretation of the CTV scans. Pure extraluminal lesion indicates no evidence of a filling defect on the CTV scan, thrombus indicates a filling defect on the CTV scan, and both indicates evidence of a filling defect and an obstructive extraluminal lesion.

If The dural venous sinuses were subdivided into the lateral system (transverse and sigmoid sinuses) and the central system (SSS, confluence of sinuses, and sinus cerebelli). When more than 1 system was involved, the more severely obstructed sinus was considered.

and signs presented by the patients in both the positiveand negative-CTV groups could be explained by the head injury itself. Nevertheless, although without statistical significance, in the positive-CTV group more patients were severely injured (GCS score $\leq 8$ on arrival) with a tendency toward a lower score on the GCS than the patients in the negative-CTV group. Moreover, within the positiveCTV group, all severely injured patients were found to have DST on their CTV scans, while most mildly to moderately injured patients had pure extraluminal obstruction $(p=0.007)$. This supports an association between the severity of injury and the formation of a thrombus within the DVS system. Moreover, 2 patients deteriorated due to sinus thrombosis: 1 patient had the signs and symptoms of increased ICP (e.g., headache, vomiting) and cranial nerve VI nerve palsy, and 1 patient had increased ICP. Both pa- tients exhibited papilledema. In both patients, anticoagulation therapy was given and improvement was noted. Currently in our practice, patients who clinically deteriorate and for whom initial imaging shows a fracture adjacent to DVS, CTV is performed to exclude occlusion of the sinuses. If a thrombus is noted, anticoagulation therapy should be considered after taking into account the risk of rebleeding. Contrasting with our findings, other researchers $^{6,19,29,36}$ did not find a correlation between the severity of the cranial trauma and the formation of DST.

As with the clinical manifestations, some radiological findings - such as intracranial air bubbles adjacent to the fracture line, contusions, and parenchymal hemorrhagesresulted from the cranial trauma itself rather than from the consequences of an obstructed DVS. Such radiological findings were detected almost equally on the NCCT scans of the patients with positive and negative CTV. On the other hand, white matter edema detected on the NCCT scans was more prevalent among patients in the positivethan the negative-CTV group $(\mathrm{p}=0.01)$. Our observed rate of white matter edema is higher than reported in the literature ${ }^{22}$ and can be explained by the different etiologies of the thrombosis. This statistically significant difference, and the higher prevalence of white matter edema among patients with a component of DST in the positiveCTV group (though this difference is not statistically significant), also suggests that an obstructed and especially thrombotic DVS has a distinct effect on the involvement of white matter edema among patients with DST. Moreover, since noncontrast CT is still the imaging technique of choice in most emergency departments, even in developed countries, all radiologists should be familiar with the early signs of sinus thrombosis. ${ }^{18}$

Among the patients in the positive-CTV group, the involvement of the TS and sigmoid sinuses was more than 3 -fold greater than that of the SSS. Importantly, patients with SSS involvement had a worse outcome $(\mathrm{p}=0.05)$, probably because of the absence of an alternative route to flow. This tendency toward greater involvement of the TS and sigmoid sinus was reported by Stiefel et al. as well: ${ }^{34}$ all 8 of their patients showed TS or sigmoid sinus thrombosis. This distribution is unique to traumatic DST. In contrast, in cohorts of patients with DST due to other causes, a tendency toward statistical equivalence between involvement of the SSS and between TS and sigmoid sinus was usually found. 7.12

CTV provides a rapid and reliable modality for detecting DST. CTV is at least equivalent to MR venography for the diagnosis of DST and is considered the method of choice in trauma cases. ${ }^{23,24,31}$ In addition, in many medical centers CTV is the only imaging technique, among the 3 mentioned above, that is available for the diagnosis of DVS obstruction in the context of head trauma. To evaluate the diagnostic value of the NCCT scan for DVS involvement among our patients, we compared its findings to those of CTV for each patient. The dense triangle sign was found on the NCCT scans of 8 patients in the positive-CTV group and in 1 patient in the negative-CTV group. NCCT has high specificity (95\%), but low sensitivity (38\%), for the detection of an obstructed DVS, with positive and negative predictive values of $89 \%$ and $59 \%$, respectively. As a 
consequence, a nonsignificant NCCT scan of head-injured patients with a fracture line adjacent to a DVS cannot rule out the presence of DVS obstruction. Therefore, further radiographic investigations with CTV should be executed to exclude DVS involvement in such patients.

As might be expected, an unfavorable outcome, as was evaluated at 1 year after the injury in the neurosurgery clinic, tended to correlate with the severity of injury with complete rather than partial obstruction and the presence of a thrombus within a DVS. However, only the latter was found to be statistically significant $(p=0.02)$. A larger prospective study is needed to determine if an occlusive process of a DVS (thrombotic or extraluminal) is clearly related to poorer outcome, or if they are both (the occlusive process and the poorer outcome) unrelated results of an initial severe injury. However, according to our clinical ex- perience with patients demonstrating posttraumatic DVS obstruction, it seems that-in addition to the major importance of the severity of the head injury itself-complete obstruction of a DVS lumen due to a thrombus formation, rather than extraluminal obstruction, has a distinct effect on outcome.

Only 2 patients in our cohort were treated specifically for the thrombotic process with anticoagulants, and both showed clinical and radiological improvement. Retrospective analyses of patients in the positive-CTV group revealed 3 additional patients among those with unfavorable outcome and 2 patients with favorable outcome, but long recovery times and moderate neurological deficits (GOS Score 4). These patients all had extensive DST and might have benefited from anticoagulant therapy. Although anticoagulation therapy was proven to be safe and effective

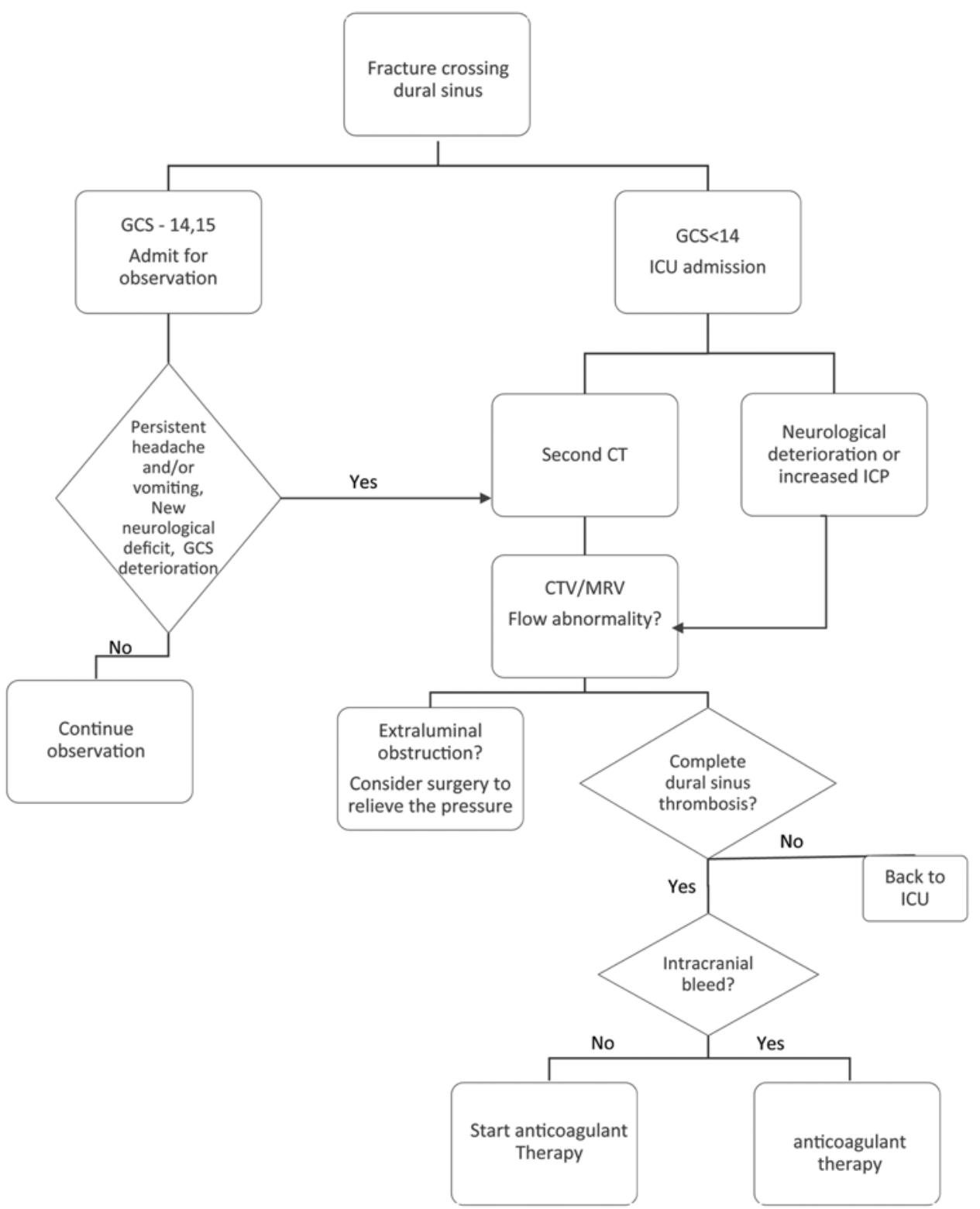

FIG. 4. A proposed flowchart for identifying patients with posttraumatic dural sinus thrombosis. MRV = magnetic resonance venography. 
for nontraumatic DST, even in the presence of intracranial hemorrhage, , 5,7,13,14 a randomized clinical study is needed to establish the safety and effectiveness of this treatment for traumatic DST.

Following our results, we established a revised protocol for patients with skull fracture crossing a dural sinus. If the patient suffers a moderate to severe head injury, we perform CTV and also a second CT, regardless of whether the patient was operated on. If the patient deteriorates, we perform CTV as soon as possible. Patients with mild head injury (GCS Scores 14-15) are observed more carefully with frequent neurological checks. If the symptoms of headache and/or vomiting persist, GCS deteriorates, or a new neurological deficit presents, we perform CTV. CTV is interpreted by a trained neuroradiologist to determine if there is flow disturbance and distinguish between thrombosis and extraluminal obstruction. If there is no flow in the sinus, then we consider the need for surgery (if there is extraluminal obstruction) or anticoagulant therapy in case of thrombosis. Figure 4 shows a proposed flowchart for identifying patients who may need treatment for a flow abnormality.

The data in our study raise several methodological issues that must be taken into account. First, the possibility of ascertainment bias needs to be considered. However, since we cross-matched data from 3 sources, 2 of which were computerized, we consider significant ascertainment bias to be unlikely. Second, the size of our cohort was not sufficient to define risk factors, either for the development of DVS obstruction in patients with a skull fracture adjacent to a DVS or for poor outcome among those who were found to have an obstructed DVS lumen (whether it was an extraluminal obstruction or DST). Nevertheless, in most cases, the more severely injured patients were followed in our institution until their neurological condition was stabilized. Third, a major limitation of our study is its retrospective design. As discussed above, further investigations are necessary to answer questions that arise from such clinical and radiological findings.

\section{Conclusions}

The presentation of some degree of DVS distortion following head injury is more frequent than reported and occurs in half of patients with skull fracture adjacent to a DVS. The severity of the head injury appears to be correlated to the potential to develop DST. Routine NCCT is not sensitive enough for the detection of distorted DVS, and CTV should be performed to rule it out in patients with skull fractures adjacent to a DVS. We showed that in most patients with an established DVS distortion, the clinical course was benign. However, the potential for a devastating outcome does exist, especially in cases with an extensive thrombotic process and when the injury involves the SSS. Further investigations are needed to define the risk factors for severe outcome and establish the optimal treatment for these patients. Finally, we suggest a separate and more precise term - traumatic dural venous sinus distortion, with or without thrombosis-for cases of DVS involvement following head injury, instead of the traditional term of dural sinus thrombosis.

\section{References}

1. Beller AJ: Benign post-traumatic intracranial hypertension. $\mathbf{J}$ Neurol Neurosurg Psychiatry 27:149-152, 1964

2. Bousser MG: Cerebral venous thrombosis: diagnosis and management. J Neurol 247:252-258, 2000

3. D'Alise MD, Fichtel F, Horowitz M: Sagittal sinus thrombosis following minor head injury treated with continuous urokinase infusion. Surg Neurol 49:430-435, 1998

4. Davis PC, Drayer BP, Anderson RE, Braffman B, Deck MD, Hasso AN, et al: Head trauma. American College of Radiology. ACR Appropriateness Criteria. Radiology 215 Suppl:507-524, 2000

5. de Bruijn SF, Stam J: Randomized, placebo-controlled trial of anticoagulant treatment with low-molecular-weight heparin for cerebral sinus thrombosis. Stroke 30:484-488, 1999

6. Delgado Almandoz JE, Kelly HR, Schaefer PW, Lev MH, Gonzalez RG, Romero JM: Prevalence of traumatic dural venous sinus thrombosis in high-risk acute blunt head trauma patients evaluated with multidetector CT venography. Radiology 255:570-577, 2010

7. deVeber G, Andrew M, Adams C, Bjornson B, Booth F, Buckley DJ, et al: Cerebral sinovenous thrombosis in children. N Engl J Med 345:417-423, 2001

8. du Plessis JJ: Depressed skull fracture involving the superior sagittal sinus as a cause of persistent raised intracranial pressure: a case report. J Trauma 34:290-292, 1993

9. Duke BJ, Ryu RK, Brega KE, Coldwell DM: Traumatic bilateral jugular vein thrombosis: case report and review of the literature. Neurosurgery 41:680-683, 1997

10. Ferrera PC, Pauze DR, Chan L: Sagittal sinus thrombosis after closed head injury. Am J Emerg Med 16:382-385, 1998

11. Ferro JM: [Cerebral venous thrombosis.] J Neuroradiol 29:231-239, 2002 (Fr)

12. Ferro JM, Canhão P, Stam J, Bousser MG, Barinagarrementeria F: Prognosis of cerebral vein and dural sinus thrombosis: results of the International Study on Cerebral Vein and Dural Sinus Thrombosis (ISCVT). Stroke 35:664-670, 2004

13. Fink JN, McAuley DL: Cerebral venous sinus thrombosis: a diagnostic challenge. Intern Med J 31:384-390, 2001

14. Greenberg M (ed): Handbook of Neurosurgery, ed 7. New York: Thieme, 2010

15. Hesselbrock R, Sawaya R, Tomsick T, Wadhwa S: Superior sagittal sinus thrombosis after closed head injury. Neurosurgery 16:825-828, 1985

16. Huisman TA, Holzmann D, Martin E, Willi UV: Cerebral venous thrombosis in childhood. Eur Radiol 11:1760-1765, 2001

17. Jennett B, Bond M: Assessment of outcome after severe brain damage. Lancet 1:480-484, 1975

18. Karthikeyan D, Vijay S, Kumar T, Kanth L: Cerebral venous thrombosis - spectrum of CT findings. Indian J Radiol Imaging 14:129-137, 2004

19. Kinal ME: Traumatic thrombosis of dural venous sinuses in closed head injuries. J Neurosurg 27:142-145, 1967

20. Klingebiel R, Busch M, Bohner G, Zimmer C, Hoffmann $\mathrm{O}$, Masuhr F: Multi-slice CT angiography in the evaluation of patients with acute cerebrovascular disease-a promising new diagnostic tool. J Neurol 249:43-49, 2002

21. Kuether TA, O’Neill O, Nesbit GM, Barnwell SL: Endovascular treatment of traumatic dural sinus thrombosis: case report. Neurosurgery 42:1163-1167, 1998

22. Leach JL, Fortuna RB, Jones BV, Gaskill-Shipley MF: Imaging of cerebral venous thrombosis: current techniques, spectrum of findings, and diagnostic pitfalls. Radiographics 26 (Suppl 1):S19-S43, 2006

23. Lee SK, terBrugge KG: Cerebral venous thrombosis in adults: the role of imaging evaluation and management. Neuroimaging Clin N Am 13:139-152, 2003

24. Linn J, Ertl-Wagner B, Seelos KC, Strupp M, Reiser M, 
Brückmann H, et al: Diagnostic value of multidetector-row $\mathrm{CT}$ angiography in the evaluation of thrombosis of the cerebral venous sinuses. AJNR Am J Neuroradiol 28:946-952, 2007

25. Meier U, Gärtner F, Knopf W, Klötzer R, Wolf O: The traumatic dural sinus injury-a clinical study. Acta Neurochir (Wien) 119:91-93, 1992

26. Ochagavia AR, Boque MC, Torre C, Alonso S, Sirvent JJ: Dural venous sinus thrombosis due to cranial trauma. Lancet 347:1564, 1996

27. Ozsvath RR, Casey SO, Lustrin ES, Alberico RA, Hassankhani A, Patel M: Cerebral venography: comparison of CT and MR projection venography. AJR Am J Roentgenol 169:1699-1707, 1997

28. Provenzale JM, Joseph GJ, Barboriak DP: Dural sinus thrombosis: findings on $\mathrm{CT}$ and MR imaging and diagnostic pitfalls. AJR Am J Roentgenol 170:777-783, 1998

29. Rivkin MA, Saraiya PV, Woodrow SI: Sinovenous thrombosis associated with skull fracture in the setting of blunt head trauma. Acta Neurochir (Wien) 156:999-1007, 2014

30. Saneto RP, Samples S, Kinkel RP: Traumatic intracerebral venous thrombosis associated with an abnormal golf swing. Headache 40:595-598, 2000

31. Saposnik G, Barinagarrementeria F, Brown RD Jr, Bushnell CD, Cucchiara B, Cushman M, et al: Diagnosis and management of cerebral venous thrombosis: a statement for healthcare professionals from the American Heart Association/ American Stroke Association. Stroke 42:1158-1192, 2011

32. Stam J, Majoie CB, van Delden OM, van Lienden KP, Reekers JA: Endovascular thrombectomy and thrombolysis for severe cerebral sinus thrombosis: a prospective study. Stroke 39:1487-1490, 2008

33. Stefini R, Latronico N, Cornali C, Rasulo F, Bollati A: Emergent decompressive craniectomy in patients with fixed dilated pupils due to cerebral venous and dural sinus thrombosis: report of three cases. Neurosurgery 45:626-630, 1999

34. Stiefel D, Eich G, Sacher P: Posttraumatic dural sinus thrombosis in children. Eur J Pediatr Surg 10:41-44, 2000
35. Stringer WL, Peerless SJ: Superior sagittal sinus thrombosis after closed head injury. Neurosurgery 12:95-97, 1983

36. Taha JM, Crone KR, Berger TS, Becket WW, Prenger EC: Sigmoid sinus thrombosis after closed head injury in children. Neurosurgery 32:541-546, 1993

37. Tsai FY, Kostanian V, Rivera M, Lee KW, Chen CC, Nguyen TH: Cerebral venous congestion as indication for thrombolytic treatment. Cardiovasc Intervent Radiol 30:675-687, 2007

\section{Disclosures}

The authors report no conflict of interest concerning the materials or methods used in this study or the findings specified in this paper.

\section{Author Contributions}

Conception and design: Shelef, Benifla, Yoel, Cohen. Acquisition of data: all authors. Analysis and interpretation of data: Shelef, Benifla, Yoel. Drafting the article: Shelef, Benifla, Yoel. Critically revising the article: Shelef, Benifla, Yoel. Reviewed submitted version of manuscript: Shelef, Benifla, Yoel, Melamed. Approved the final version of the manuscript on behalf of all authors: Shelef. Statistical analysis: Shelef, Benifla, Yoel. Administrative/ technical/material support: Shelef, Benifla, Yoel, Merkin, Cohen. Study supervision: Shelef, Benifla, Yoel, Cohen.

\section{Supplemental Information \\ Previous Presentations}

The paper was presented at the 2010 Israeli Neurosurgical Annual meeting in Jerusalem.

\section{Correspondence}

Ilan Shelef, Department of Radiology, Soroka University Medical Center, Beer-Sheva 84101, Israel. email: shelef@bgu.ac.il. 